Journal of Statistical Physics, Vol. 121, Nos. 5/6, December 2005 (C 2005)

DOI: $10.1007 / \mathrm{s} 10955-005-7009-\mathrm{y}$

\title{
Chaotic Dynamics in an Electronic Model of a Genetic Network
}

\author{
Leon Glass, ${ }^{1}$ Theodore J. Perkins, ${ }^{2}$ Jonathan Mason, ${ }^{3}$ \\ Hava T. Siegelmann, ${ }^{4}$ and Roderick Edwards ${ }^{5}$
}

Received January 17, 2005; accepted June 10, 2005

\begin{abstract}
We consider dynamics in a class of piecewise-linear ordinary differential equations and in an electronic circuit that model genetic networks. In these models, gene activity varies continuously in time. However, as in Boolean or discrete-time switching networks, gene activity is driven high or low based only on whether the activities of the regulating genes are high or low (i.e., above or below certain thresholds). Depending on the "regulatory logic", these models can exhibit simple dynamics, like stable fixed points or oscillation, or chaotic dynamics. The observed qualitative and quantitative differences between the dynamics in the idealized equations and the dynamics in the electronic circuit lead us to focus attention on the analysis of the dynamics as a function of parameter values. We propose new techniques for solving the inverse problem - the problem of inferring the regulatory logic and parameters from time series data. We also give new symbolic and statistical methods for characterizing dynamics in these networks.
\end{abstract}

KEY WORDS: genetic networks; symbolic dynamics; chaos; limit cycle oscillation; inverse problem.

In honor of Mitchell Feigenbaum on his 60th birthday.

${ }^{1}$ Centre for Nonlinear Dynamics in Physiology and Medicine, Department of Physiology, McGill University, 3655 Promenade Sir William Osler, Montreal, Quebec, Canada H3G 1Y6; e-mail: glass@cnd.mcgill.ca

${ }^{2} \mathrm{McGill}$ Centre for Bioinformatics, McGill University, 3775 University Street, Montreal, Quebec, Canada H3A 2B4.

${ }^{3}$ The Krasnow Institute for Advanced Study, Mail Stop 2A1, George Mason University, Fairfax VA 22030, USA.

${ }^{4}$ Computer Science Building, 140 Governors Drive, University of Massachusetts, Amherst, MA 01003-9264, USA.

${ }^{5}$ Department of Mathematics and Statistics, University of Victoria, P.O.Box 3045, STN CSC, Victoria, BC, Canada V8W 3P4. 


\section{INTRODUCTION}

In the past decade there have been revolutionary advances in our knowledge of the DNA sequences of a large number of different organisms. ${ }^{(1)}$ Yet, knowledge of these sequences alone is not adequate to determine or understand the multiple developmental and physiological processes that underlie normal and abnormal dynamics in biological systems. One of the fundamental issues related to the functioning of genetic networks involves the control of gene expression. Early papers from Jacob and Monod proposed that different cellular dynamic behaviors, such as multiple attractors or oscillations, might arise as a consequence of the differential expression of genes. ${ }^{(2)}$ In recent years synthetic genetic networks in bacteria have been designed to display bistability and oscillations, ${ }^{(3-5)}$ proving that in principle, dynamics in genetic networks might play the roles envisioned by Jacob and Monod.

An early step towards placing the dynamics of genetic networks in a theoretical context was taken by Kauffman, who proposed that genetic networks could be modeled by randomly constructed discrete Boolean switching networks, now called Kauffman networks. ${ }^{(6,7)}$ The logical elements in the networks were the genes. The inputs to each logical element represented the proteins, called transcription factors, that regulate the genetic activity. Since the transcription factors in turn are coded for by genes, a complex network results, in which the attractors correspond to different cell types in the organism. Kauffman networks with two inputs per gene have a comparatively small number of attractors. In further studies of Kauffman networks, statistical physicists have computed the numbers of attractors as a function of network size and connectivity, ${ }^{(8,9)}$ developed criteria for irregular dynamics, ${ }^{(10,11)}$ and studied the effects of noise. ${ }^{(12)}$ However, cells in the body do not have clocking devices that can update the states of all genes simultaneously, as required by the Kauffman model. Consequently, we believe it is more appropriate to analyze dynamics in differential equation models of genetic networks that preserve the logical structure, and have carried out extensive studies in this area, ${ }^{(13-22)}$ for reviews see refs. 23 and 24 . For any Boolean switching network, there is a class of differential equations that have the same underlying structure, but whose dynamics can depend on the values of parameters such as decay constants, thresholds, and production rates.

In the following we study complex dynamics in an electronic circuit that models a class of differential equations proposed to capture qualitative aspects of the structure and dynamics of genetic networks. By analyzing a real circuit, rather than an idealized equation, we focus on techniques that in

principle could be applied to real biological systems when sufficiently precise data becomes available. 
In Section 2 we present the network equations, and summarize results from our earlier work. We also describe a hybrid analog-digital electronic circuit that simulates the differential equations. ${ }^{(25)}$ We study a five-element circuit that displays regular stable oscillations and a second circuit that displays irregular behavior suggestive of chaotic dynamics. In Section 3, we study the inverse problem, i.e., the determination of the underlying equations based on observation of the dynamics in the electronic circuit, extending earlier work on the idealized equations. ${ }^{(22)}$ The inverse problem consists of a determination of the logic of the circuit as well as the parameters. This leads to the recognition that the time constants of the different elements of the network are different. Section 4 develops theoretical methods applicable to study the dynamics in the circuit. We consider methods based on analytic computation of the return maps in these systems, with particular focus on the problems introduced by the variations in the time constants. We also propose methods for measuring the complexity of the dynamics based on symbolic representations.

\section{A MODEL GENETIC NETWORK}

\subsection{Differential Equations}

A gene is a sequence of DNA that codes for a sequence of amino acids that constitute a protein (see ref. 1 for more details on molecular biology). Even though all cells have the DNA code to make a vast array of different proteins, a variety of regulatory mechanisms, still not completely understood, determine which proteins will be synthesized in each cell. One mode of gene regulation is through a class of proteins called transcription factors. Transcription factors bind to the DNA, turning "on" or "off" the synthesis of specific mRNA molecules. These are in turn translated into proteins, some of which may be transcription factors and thus may influence mRNA synthesis. We model this dynamical system in a simplified way by letting $x_{i}$ represent the concentration of chemical species $i$ in a cell. The time rate of change of $x_{i}$ is

$$
\frac{d x_{i}}{d t}=h_{i}(\mathbf{x})-\frac{x_{i}}{\tau_{i}}, \quad i=1, \ldots, N
$$

where there are $N$ chemical species, $\mathbf{x}$ is a vector giving their concentrations, $h_{i}$ is a function giving the control of the synthesis of the $i$ th chemical species by the others, and $\tau_{i}$ is a decay constant. Differential equation models of biochemical and genetic networks are often of this form (for example, see, refs. 3-5, 13, 14 and 26-29). 
In what follows, we adopt a highly simplified but nonlinear form for the functions $h_{i}$. To each continuous variable $x_{i}(t)$, we associate a discrete variable $X_{i}(t)$,

$$
X_{i}(t)=0 \quad \text { if } x_{i}(t)<\theta_{i}, \quad \text { otherwise } X_{i}(t)=1,
$$

where $\theta_{i}$ is an arbitrary real-valued threshold. The key restriction we place on Eq. (1) is that the synthesis rates depend only on discrete states of chemical species.

$$
\frac{d x_{i}}{d t}=f_{i}(\mathbf{X}(t))-\frac{x_{i}}{\tau_{i}}, \quad i=1, \ldots, N
$$

where $\mathbf{X}(t)$ is the Boolean vector of discrete states of the chemical species at time $t$. In the biochemical context, in which the $x_{i}$ are meant to represent concentrations, we assume all $x_{i}$ are nonnegative and all $\tau_{i}$ and $\theta_{i}$ are positive. In general, however, we need not restrict the ranges of the $x_{i}$ or $\theta_{i}$, and we refer to the $x_{i}$ as elements in an interacting network.

Equation (3) is piecewise linear in $\mathbf{x}$, with the pieces defined by the threshold hyperplanes $x_{i}=\theta_{i}$, and hence is easily integrated. As long as variables cross threshold hyperplanes transversally, there will be a finite or countably-infinite sequence of switching times, $\left\{t_{1}, t_{2}, t_{3}, \ldots\right\}$, at which some element of the network crosses its threshold. We can obtain the solution of Eq. (3) for each variable $x_{i}$ for $t_{j}<t<t_{j+1}$ :

$$
x_{i}(t)=x_{i}\left(t_{j}\right) e^{-\left(t-t_{j}\right) / \tau_{i}}+\tau_{i} f_{i}(\mathbf{X}(t))\left(1-e^{-\left(t-t_{j}\right) / \tau_{i}}\right) .
$$

Thus, for any point in the orthant defined by the Boolean vector $\mathbf{X}$, element $i$ asymptotically approaches $\tau_{i} f_{i}(\mathbf{X})$. We call the vector of these values the focal point for the orthant. If the focal point is within the orthant, then it is an asymptotically stable fixed point. If it is within another orthant, then some variable will cross its threshold, and the trajectory continues in the adjacent orthant. We assume that for all $\mathbf{X}, \tau_{i} f_{i}(\mathbf{X}) \neq \theta_{i}$, so that no focal points lie on threshold hyperplanes.

The flow is therefore piecewise focused, with the focal points depending in general on the current orthant. As a consequence of variables crossing thresholds, the logical state changes and so might the focal point that the solution approaches. It is possible to piece together the trajectories and determine the dynamics for future times once the logical structure of the network and the parameters are known. 
The logical structure of the differential equation can be represented as a directed graph on an $N$-dimensional hypercube ( $N$-cube). The $N$-cube has $2^{N}$ vertices, each corresponding to a logical state, $\mathbf{X}$, of the network and hence to a region of state space. A directed edge between two adjacent vertices indicates a change in the logical state of the network that can be observed from some initial condition. Thus, edges in this representation represent flows across the boundaries of adjacent orthants of state space. The orientations of the directed edges in the graph can be determined by checking, for each $\mathbf{X}$ and $i$, whether $\tau_{i} f_{i}(\mathbf{X})$ is greater than or less than $\theta_{i}$.

Alternatively, the logical structure can be represented by a $2^{N} \times 2^{N}$ adjacency matrix, $W$, in which $w_{i j}=1$ if there is a directed edge from vertex $i$ to vertex $j$; otherwise $w_{i j}=0$.

In what follows we assume that for all $i$

$$
\operatorname{sign}\left(\tau_{i} f_{i}(\mathbf{X})-\theta_{i}\right) \text { is independent of } X_{i} .
$$

This condition holds, for example, if there is no self input, i.e., if $f_{i}$ is completely independent of $X_{i}$. If Eq. (5) holds, there is a directed edge in precisely one of the two possible directions for every pair of adjacent vertices and flows are transverse across threshold hyperplanes. Except for unusual circumstances that we do not consider in this paper, only one element crosses its threshold at a time. Consequently at each switch time precisely one element in the logical state of the network changes. In networks where Eq. (5) does not hold, more complex behaviors are possible, and Eq. (3) may need to be treated as a system of differential inclusions. ${ }^{(29)}$

\subsection{Hybrid Electronic Circuit}

To illustrate some of the properties of Eq. (3) and to set the focus for the remainder of this paper, we present an electronic circuit ${ }^{(25)}$ that simulates the properties of Eq. (3) for a network with $N=5$. When $N=5$, there are of the order of $3 \times 10^{20}$ different possible logical structures for these networks. ${ }^{(19)}$ Each of these logical structures further defines a family of equations that can be generated by varying the $\tau_{i}, \theta_{i}$ and $f_{i}$. By constructing a hybrid digital-analog circuit we can rapidly change the logic of the network.

Figure 1 gives a schematic diagram for element 5 of the circuit. We briefly describe its operation but for more details see ref. 25 . To model the regulation of genes, we apply methods of combinatorial switching circuit $\operatorname{design}^{(30)}$ using CMOS logic. This allows us to rapidly change the logical functions that control the regulation of the "on-off" states of the genes. 


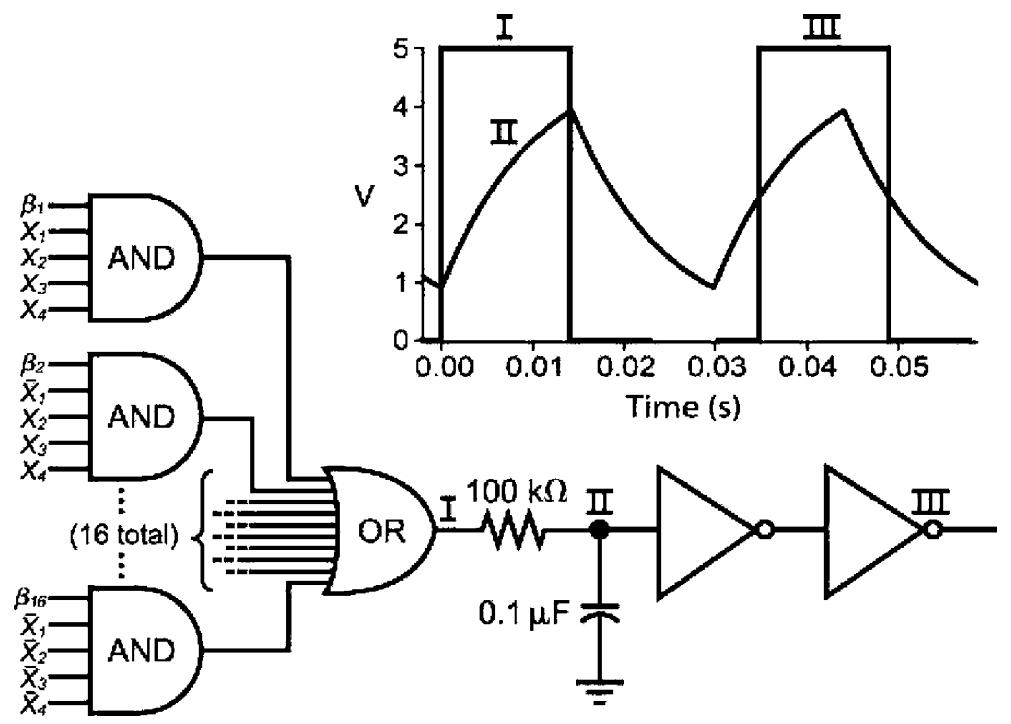

Fig. 1. A schematic diagram of one element of the electronic circuit.

Any of the $2^{16}$ logic functions of four variables can be synthesized by an appropriate combination of the control lines $\beta_{1}, \beta_{2}, \ldots, \beta_{16}$. In order to generate any logical function, the $\beta_{i}$ are combined in an AND function with all possible logical states of the four model genes that are inputs to gene 5. The output at point $\mathrm{I}$ in the circuit is thus either high or low, nominally $5 \mathrm{~V}$ or $0 \mathrm{~V}$ (see inset), and corresponds to $f_{i}(\mathbf{X}(t))$ in Eq. (3). This output is fed into an $R C$ circuit, so that the voltage at point II is given by

$$
V(t)=E+(V(0)-E) e^{-\frac{t}{R C}} .
$$

Thus, the voltage across the capacitor is an exponential function that approaches $E$ with a time constant equal to $R C$. In this sense, the voltage $V(t)$ is analogous to the concentration of a protein transcription factor $x_{i}(t)$. Moreover, we can pass the voltage through a threshold element to provide a logical variable analogous to the logical variable $X_{i}(t)$, point III in the circuit. This voltage is then fed back into the input lines for the other elements in the circuit. By selecting $R=100 \mathrm{k} \Omega$ and $C=0.1 \mu \mathrm{F}$, we set the time constant to be $\approx 10 \mathrm{~ms}$.

To illustrate the operation of the circuit and some of the properties of the differential equation, we present the output from two different choices of the logical function. The first choice is a negative feedback circuit, in 
Table I. Truth Table for the Oscillator: the Parentheses are for Section 3

\begin{tabular}{|c|c|c|c|c|c|c|c|c|c|}
\hline$X_{1}$ & $X_{2}$ & $X_{3}$ & $X_{4}$ & $X_{5}$ & $F_{1}$ & $F_{2}$ & $F_{3}$ & $F_{4}$ & $F_{5}$ \\
\hline 0 & 0 & 0 & 0 & 0 & 1 & 0 & 0 & 0 & 0 \\
\hline 0 & 0 & 0 & 0 & 1 & 0 & 0 & 0 & 0 & 0 \\
\hline 0 & 0 & 0 & 1 & 0 & (1) & (0) & $(0)$ & $(0)$ & (1) \\
\hline 0 & 0 & 0 & 1 & 1 & 0 & 0 & 0 & 0 & 1 \\
\hline 0 & 0 & 1 & 0 & 0 & (1) & $(0)$ & $(0)$ & (1) & $(0)$ \\
\hline 0 & 0 & 1 & 0 & 1 & (0) & (0) & (0) & (1) & (0) \\
\hline 0 & 0 & 1 & 1 & 0 & (1) & (0) & (0) & (1) & (1) \\
\hline 0 & 0 & 1 & 1 & 1 & 0 & 0 & 0 & 1 & 1 \\
\hline 0 & 1 & 0 & 0 & 0 & (1) & $(0)$ & (1) & $(0)$ & (0) \\
\hline 0 & 1 & 0 & 0 & 1 & (0) & (0) & (1) & (0) & (0) \\
\hline 0 & 1 & 0 & 1 & 0 & (1) & (0) & (1) & (0) & (1) \\
\hline 0 & 1 & 0 & 1 & 1 & (0) & (0) & (1) & (0) & (1) \\
\hline 0 & 1 & 1 & 0 & 0 & (1) & (0) & (1) & (1) & (0) \\
\hline 0 & 1 & 1 & 0 & 1 & (0) & (0) & (1) & (1) & (0) \\
\hline 0 & 1 & 1 & 1 & 0 & (1) & (0) & (1) & (1) & (1) \\
\hline 0 & 1 & 1 & 1 & 1 & 0 & 0 & 1 & 1 & 1 \\
\hline 1 & 0 & 0 & 0 & 0 & 1 & 1 & 0 & 0 & 0 \\
\hline 1 & 0 & 0 & 0 & 1 & $(0)$ & (1) & $(0)$ & $(0)$ & (0) \\
\hline 1 & 0 & 0 & 1 & 0 & (1) & (1) & (0) & (0) & (1) \\
\hline 1 & 0 & 0 & 1 & 1 & (0) & (1) & (0) & (0) & (1) \\
\hline 1 & 0 & 1 & 0 & 0 & (1) & (1) & (0) & (1) & (0) \\
\hline 1 & 0 & 1 & 0 & 1 & (0) & (1) & (0) & (1) & (0) \\
\hline 1 & 0 & 1 & 1 & 0 & (1) & (1) & (0) & (1) & (1) \\
\hline 1 & 0 & 1 & 1 & 1 & (0) & (1) & (0) & (1) & (1) \\
\hline 1 & 1 & 0 & 0 & 0 & 1 & 1 & 1 & 0 & 0 \\
\hline 1 & 1 & 0 & 0 & 1 & $(0)$ & (1) & (1) & $(0)$ & (0) \\
\hline 1 & 1 & 0 & 1 & 0 & (1) & (1) & (1) & (0) & (1) \\
\hline 1 & 1 & 0 & 1 & 1 & (0) & (1) & (1) & (0) & (1) \\
\hline 1 & 1 & 1 & 0 & 0 & 1 & 1 & 1 & 1 & 0 \\
\hline 1 & 1 & 1 & 0 & 1 & $(0)$ & (1) & (1) & (1) & (0) \\
\hline 1 & 1 & 1 & 1 & 0 & 1 & 1 & 1 & 1 & 1 \\
\hline 1 & 1 & 1 & 1 & 1 & 0 & 1 & 1 & 1 & 1 \\
\hline
\end{tabular}

which gene $k$ stimulates gene $k+1$ for $k=1,2,3,4$, but gene 5 inhibits gene 1. The truth table is shown in Table I. Based on Example 3 on p. 216 in ref. 16 (see also the discussion in Section 4.1), we expect that this network will display a stable limit cycle oscillation, Fig. 2(a). In computing the trajectory in Fig. 2(a), we assume that the voltage $V(t)$ in Eq. (6) asymptotically approaches $5 \mathrm{~V}$ or $0 \mathrm{~V}$ (corresponding to a 1 or 0 , respectively, in the truth table entry for $\mathbf{X}(t)$ in Table I), and that $\theta_{i}=2.5 \mathrm{~V}$ and $\tau_{i}=10 \mathrm{~ms}$ for all $i$. 
Table II. Truth Table for the Complex Network: the Parentheses and Brackets are for Section 3

\begin{tabular}{|c|c|c|c|c|c|c|c|c|c|}
\hline$X_{1}$ & $X_{2}$ & $X_{3}$ & $X_{4}$ & $X_{5}$ & $F_{1}$ & $F_{2}$ & $F_{3}$ & $F_{4}$ & $F_{5}$ \\
\hline 0 & 0 & 0 & 0 & 0 & 0 & 0 & 0 & 1 & 1 \\
\hline 0 & 0 & 0 & 0 & 1 & 0 & 1 & 1 & 1 & 1 \\
\hline 0 & 0 & 0 & 1 & 0 & 0 & 0 & 1 & 1 & 1 \\
\hline 0 & 0 & 0 & 1 & 1 & 0 & 1 & 0 & 1 & 1 \\
\hline 0 & 0 & 1 & 0 & 0 & 0 & 1 & 0 & 1 & 0 \\
\hline 0 & 0 & 1 & 0 & 1 & 0 & 0 & 1 & 1 & 0 \\
\hline 0 & 0 & 1 & 1 & 0 & 1 & 0 & 1 & 1 & 0 \\
\hline 0 & 0 & 1 & 1 & 1 & 1 & 1 & 0 & 1 & 0 \\
\hline 0 & 1 & 0 & 0 & 0 & {$[0]$} & 0 & 0 & 0 & 1 \\
\hline 0 & 1 & 0 & 0 & 1 & 1 & 1 & 1 & 0 & 1 \\
\hline 0 & 1 & 0 & 1 & 0 & (1) & $(0)$ & (1) & $(0)$ & (0) \\
\hline 0 & 1 & 0 & 1 & 1 & 0 & 1 & 1 & 0 & 0 \\
\hline 0 & 1 & 1 & 0 & 0 & 1 & 1 & 0 & 0 & 1 \\
\hline 0 & 1 & 1 & 0 & 1 & 0 & 0 & 1 & 0 & 1 \\
\hline 0 & 1 & 1 & 1 & 0 & {$[0]$} & 0 & 1 & 0 & 0 \\
\hline 0 & 1 & 1 & 1 & 1 & 1 & 1 & 1 & 0 & 0 \\
\hline 1 & 0 & 0 & 0 & 0 & 0 & 0 & 0 & 1 & 0 \\
\hline 1 & 0 & 0 & 0 & 1 & 0 & 0 & 0 & 1 & 0 \\
\hline 1 & 0 & 0 & 1 & 0 & 0 & 0 & 0 & 1 & 1 \\
\hline 1 & 0 & 0 & 1 & 1 & 0 & [1] & 1 & 1 & 1 \\
\hline 1 & 0 & 1 & 0 & 0 & 0 & 0 & 0 & 0 & 0 \\
\hline 1 & 0 & 1 & 0 & 1 & 0 & 1 & 0 & 0 & 0 \\
\hline 1 & 0 & 1 & 1 & 0 & 1 & 0 & 0 & 0 & 1 \\
\hline 1 & 0 & 1 & 1 & 1 & 1 & 0 & 1 & 0 & 1 \\
\hline 1 & 1 & 0 & 0 & 0 & {$[0]$} & 0 & 0 & 0 & 1 \\
\hline 1 & 1 & 0 & 0 & 1 & 1 & 0 & 0 & 0 & 1 \\
\hline 1 & 1 & 0 & 1 & 0 & (1) & $(0)$ & $(0)$ & $(0)$ & (0) \\
\hline 1 & 1 & 0 & 1 & 1 & (0) & (1) & (1) & (0) & (0) \\
\hline 1 & 1 & 1 & 0 & 0 & 1 & 0 & 0 & 0 & 1 \\
\hline 1 & 1 & 1 & 0 & 1 & 0 & 1 & 0 & 0 & 1 \\
\hline 1 & 1 & 1 & 1 & 0 & $(0)$ & $(0)$ & $(0)$ & $(0)$ & (0) \\
\hline 1 & 1 & 1 & 1 & 1 & (1) & (0) & (1) & (0) & (0) \\
\hline
\end{tabular}

The quantitative differences in the dynamics between the idealized case and the actual circuit, Fig. 2(b), reflect differences in the time constants, thresholds, and asymptotic values that are introduced by the actual workings of the circuit. However, the observation of a stable oscillation with a similar waveform poses theoretical questions concerning the stability and classification of the limit cycle oscillation. The discussion of these issues is the focus of Section 4.1. 

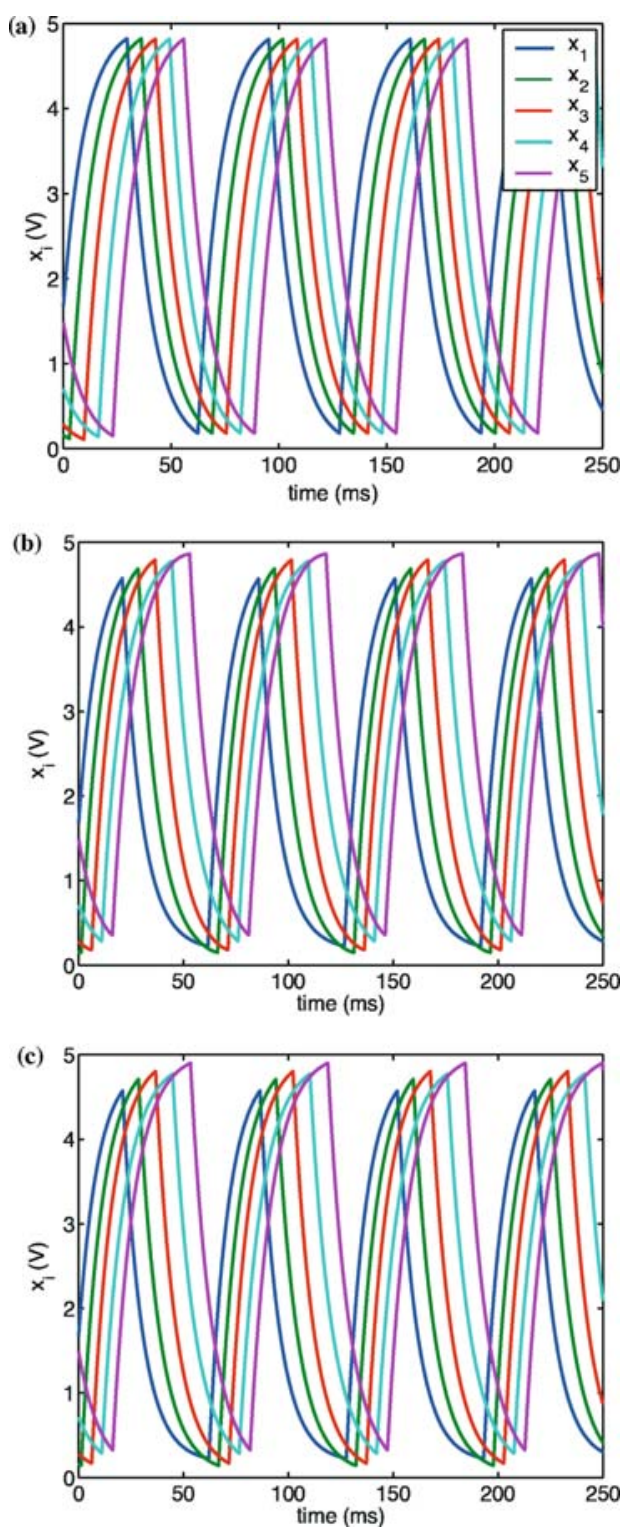

Fig. 2. Dynamics in the feedback inhibition loop of five variables given by the truth table in Table I. (a) The dynamics in the differential equation with all $\tau_{i}=10 \mathrm{~ms}, \theta_{i}=2.5 \mathrm{~V}$ and assuming asymptotic approach to $5 \mathrm{~V}$, if an element is on, or $0 \mathrm{~V}$, if an element is off. (b) The dynamics in the electronic circuit. (c) The dynamics in the differential equation using parameter values determined in the text (see Section 3). 
As a second network, we consider the truth table in Table II. The results of integrating this network with focal point coordinates at $5 \mathrm{~V}$ and $0 \mathrm{~V}, \tau_{i}=$ $10 \mathrm{~ms}, \theta_{i}=2.5 \mathrm{~V}$ are shown in Fig. 3(a). Following the transient which is still present in the figure, the trajectory approaches a stable limit cycle oscillation with period $\approx 60.8 \mathrm{~ms}$. The operation of the electronic circuit generates an extremely complex waveform, Fig. 3(b). Although the idealized equations show a stable limit cycle with complex waveform, the circuit generates complex aperiodic dynamics. In Sections 4.2 and 4.3, we discuss theoretical issues posed by this network, and discuss symbolic methods that can be used to characterize the dynamics. Although we are not able to prove chaotic dynamics for the electronic circuit, the symbolic dynamic analysis of the differential equation that we present in Sections 4.2 and 4.3 is consistent with the conjecture that this network displays deterministic chaos characterized by aperiodic dynamics with sensitive dependence on initial conditions.

\section{THE INVERSE PROBLEM}

The inverse problem for gene regulatory networks is to estimate regulatory logic and parameters from observed dynamical behavior. In earlier work, we showed that this was possible under idealized conditions and estimated the amount of time series data needed. ${ }^{(22)}$ DeJong and colleagues have manually inferred the regulatory logic for several genetic network models of the type Eq. (3). ${ }^{(29)}$ They do not have an automated technique, however, and they do not fit the quantitative parameters. Here, we focus on solving the inverse problem for time series from the electronic network. We make the simplifying assumption that for each element $i, \tau_{i} f_{i}$ takes only one of two values, which we denote $z_{i}^{0}$ and $z_{i}^{1}$. These two correspond to the low and high focal point coordinates for element $i$, respectively. As such, the inverse problem is to estimate the $z_{i}^{0}, z_{i}^{1}, \tau_{i}, \theta_{i}$ and $f_{i}$ from time series data of the form $\mathbf{x}\left(t_{1}\right), \mathbf{x}\left(t_{2}\right), \mathbf{x}\left(t_{3}\right), \ldots$ We describe an approach that is successful on the oscillator time series. The complex time series is discussed at the end of the section.

To infer $z_{i}^{0}, z_{i}^{1}$ and $\tau_{i}$ for each element we select two intervals from the oscillator time series - one in which $x_{i}$ is clearly rising and one in which $x_{i}$ is clearly falling. For any choice of $\tau_{i}$, the choices of $z_{i}^{0}$ and $z_{i}^{1}$ that minimize the mean squared error between predicted and observed $x_{i}$ values can be determined analytically. To optimize $\tau_{i}$, we simply test all possibilities in the finite set $5.00 \mathrm{~ms}, 5.01 \mathrm{~ms}, \ldots, 15.00 \mathrm{~ms}$. The range includes the expected value of about $10 \mathrm{~ms}$. 

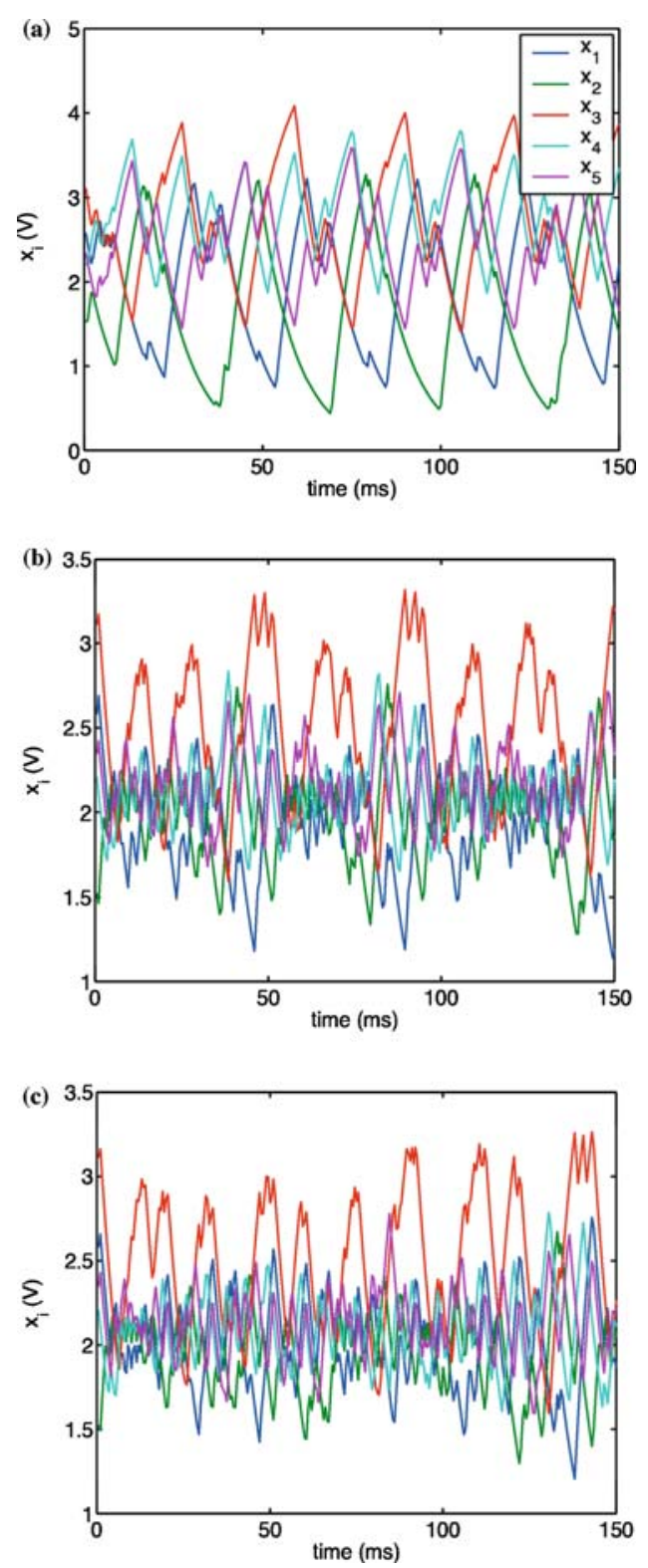

Fig. 3. Dynamics in the complex network given by the truth table in Table 2. (a) The dynamics in the differential equation with all $\tau_{i}=10 \mathrm{~ms}, \theta_{i}=2.5 \mathrm{~V}$ and assuming asymptotic approach to $5 \mathrm{~V}$, if an element is on, or $0 \mathrm{~V}$, if an element is off. (b) The dynamics in the electronic circuit. (c) The dynamics in the differential equation using parameter values determined in the text, see Section 3. 
For a given choice of $\theta_{i}$, we can estimate $f_{i}$ as follows. First, tentatively assign $x_{i}\left(t_{j}\right)$ to be rising if the forward and backward differences are positive. Assign it to be falling if both differences are negative. Now consider a particular Boolean vector $\mathbf{X}$. Find all time points at which the logical inputs to $f_{i}$, based on the given choice of $\theta_{i}$, are $\mathbf{X}$. If at these time points, a tentative assignment of rising is more common than a tentative assignment of falling, estimate $f_{i}(\mathbf{X})=z_{i}^{1} / \tau_{i}$. If falling is more common, then let $f_{i}(\mathbf{X})=z_{i}^{0} / \tau_{i}$. Otherwise, let $f_{i}(\mathbf{X})=$ "'?".

To estimate the $\theta_{i}$ we use a local search procedure. We evaluate a set of $\theta_{i}$ by estimating the $f_{i}$ as above, based on $\theta_{i}$, and simulating the dynamics starting from each $\mathbf{x}\left(t_{j}\right)$ for time $t_{j+1}-t_{j}$. We sum the squared error between the simulated $\mathbf{x}\left(t_{j+1}\right)$ and the observed $\mathbf{x}\left(t_{j+1}\right)$. Preliminary analysis indicated that the thresholds $\theta_{i} \approx 2.1 \mathrm{~V}$. Therefore, we start with all $\theta_{i}=2.1 \mathrm{~V}$, and we repeatedly find the single change of $\pm 0.001 \mathrm{~V}$ to any $\theta_{i}$, which most reduces the sum of squared errors. The search ends at a local minimum. Using this approach, we estimate the following parameters based on the oscillator data.

\begin{tabular}{llllll}
\hline & $i=1$ & \multicolumn{1}{c}{2} & \multicolumn{1}{c}{3} & \multicolumn{1}{c}{4} & \multicolumn{1}{c}{5} \\
\hline$\theta_{i}(\mathrm{~V})$ & 2.084 & 2.077 & 2.092 & 2.128 & 2.073 \\
$z_{i}^{1}(\mathrm{~V})$ & 4.895 & 5.009 & 5.006 & 4.952 & 5.021 \\
$z_{i}^{0}(\mathrm{~V})$ & 0.179 & 0.048 & 0.045 & 0.067 & 0.032 \\
$\tau_{i}(\mathrm{~ms})$ & 9.08 & 9.79 & 9.59 & 10.33 & 10.12 \\
\hline
\end{tabular}

The logic inferred by this approach is indicated in Table I. Correctly inferred logic is indicated in regular type and logic that could not be inferred is indicated by parentheses. No inference was possible for many of the logical network states because those states never occurred in the time series. There were no incorrect inferences. Figure 2(c) shows the simulated dynamics resulting from these parameters. They match the observed dynamics closely.

It is more difficult to extract network parameters from the complex time series. The sampling frequency and switching frequency are close enough that it is difficult to estimate when elements are rising and when they are falling, a prerequisite of our technique. However, by manually selecting and fitting multiple stretches of the time series in which an element appears to be rising or falling, reasonable estimates of the $z_{i}^{0}, z_{i}^{1}$ and $\tau_{i}$ can be made. It is also possible to get most of the logic correct simply by assuming $\theta_{i}=2.1 \mathrm{~V}$ for all elements. Table II shows the correctly 
inferred logic in regular type. Incorrect inferences are indicated by square brackets (with the correct value displayed), and logic which could not be inferred is indicated in parentheses. Most logical network states appeared in the time series, allowing nearly the whole truth table to be inferred. Figure 3(c) shows the simulated dynamics when using the correct logic for the complex network and the quantitative parameter estimates based on the oscillator data (as opposed to those based on the complex time series). The simulation in Fig. 3(c) appears to capture the observed time series in Fig. 3(b) better than the simulation using the idealized parameters in Fig. 3(a).

\section{THEORETICAL ANALYSIS OF THE NETWORK DYNAMICS}

Because of its piecewise-linear nature and logical structure, Eq. (3) is amenable to theoretical analysis. ${ }^{(16,23,24)}$ A motivating question in our work is to make statements about the dynamics in Eq. (3) based simply on the truth table for the network, as reflected in the associated directed graph on the $N$-cube showing allowed transitions between neighboring orthants. We now discuss theoretical methods that use the $N$-cube representation to help determine properties of the dynamics based on computation of the Poincare map (Section 4.1) and using symbolic methods (Sections 4.2 and 4.3).

\subsection{Analytic Computation of the Poincaré Map}

In certain special cases, knowledge about the dynamics in the differential equation follows immediately from the $N$-cube representation. A stable vertex on the $N$-cube is a vertex with no outgoing edges. A cycle is a directed path on the $N$-cube that starts and ends on the same vertex. A cyclic attractor is a cycle on the $N$-cube in which each vertex on the cycle has $N-1$ edges directed towards it, and one edge directed away from it. If an $N$-cube has no cycles, then in the limit $t \rightarrow \infty$, solutions of Eq. (3) approach a stable fixed point corresponding to one of the stable vertices in the $N$-cube. Which vertex is reached depends on the initial condition when there is more than one stable fixed point.

A cycle on the $N$-cube is a necessary condition for a cycle in Eq. (3). For situations in which there are cycles, it is useful to compute the Poincaré map describing the return to a hyperplane between two adjacent orthants.

If all the decay constants $\tau_{i}$ are equal, the maps that take the flows from one orthant boundary to the next have a simple form called a linear 
fractional map

$$
M(\mathbf{x})=\frac{A \mathbf{x}}{1+\langle\phi, \mathbf{x}\rangle},
$$

where the thresholds are translated to zero, $\mathbf{x} \in \mathbf{R}^{N}$ is a point on the initial orthant boundary (in an $N$-dimensional network), $A$ is an $N \times N$ matrix, $\phi \in \mathbf{R}^{N}$, and $\langle\phi, \mathbf{x}\rangle$ represents a vector dot product between $\phi$ and $\mathbf{x}$. This form for $\mathrm{Eq}$. (7) follows directly from the solution of Eq. (4), where $A$ and $\phi$ depend on the focal point coordinates $f_{i}$ of the flow for the orthants being traversed. The composition of two linear fractional maps of the same dimension is once again a linear fractional map. As a consequence of this property, if there is a cycle we can analytically (usually with the assistance of a computer) compute the return map for a given cycle of orthants starting on a particular orthant boundary crossing. The return map is often called the Poincaré map, which can be represented as in Eq. (7), but where $A$ is an $(N-1) \times(N-1)$ matrix, and $\phi$ and $\mathbf{x}$ are $(N-1)$ vectors. $^{(23)}$

If the directed graph on the $N$-cube displays a cyclic attractor, by a change of coordinates we can represent $A$ as a positive matrix and $\phi$ as a positive vector. The limiting dynamics of Eq. (7) under iteration can be analyzed by application of the Perron-Frobenius theorem. ${ }^{(16)}$ If the leading eigenvalue of $A$ is greater than 1, the iteration of the map converges to a unique stable fixed point corresponding to a unique stable limit cycle oscillation in the differential equation. Many other cycles are not cyclic attractors but are nevertheless locally stable and the Perron-Frobenius theorem still applies. ${ }^{(23)}$

The five-dimensional feedback inhibition network, represented by the truth table in Table I, has the cyclic attractor: $00000 \rightarrow 10000 \rightarrow 11000 \rightarrow$ $11100 \rightarrow 11110 \rightarrow 11111 \rightarrow 01111 \rightarrow 00111 \rightarrow 00011 \rightarrow 00001 \rightarrow 00000 \ldots$ For the idealized case in which all time constants are equal to 1 , all the focal point coordinates are at $5 \mathrm{~V}$ and $0 \mathrm{~V}$, and all the thresholds are $2.5 \mathrm{~V}$, the leading eigenvalue of $A$ is greater than 1 , and there is a unique stable limit cycle oscillation. Further, the period of the oscillation is given by $-10 \ln w$, where $w$ is the unique root of the equation $w^{4}+w^{3}+w^{2}+w-$ $1=0$ in the interval $0<w<1$. $^{(15)}$ Given the $10 \mathrm{~ms}$ time constant in this circuit, we therefore predict that the period is $\approx 65.56256 \ldots \mathrm{ms}$, Fig. 2(a). The actual dynamics in this circuit are shown in Fig. 2(b). There is a stable oscillation with a period of $\approx 65.4896 \mathrm{~ms}$, close to the theoretical value.

This analysis depends on the explicit computation and analysis of the Poincaré map, and this is only possible for the case in which all the time constants are equal. The stable cycle observed for the circuit leads us to 
conjecture that, just as in the case in which all time constants are equal, for networks displaying cyclic attractors based on their logical structure, independent of the values of the time constants the dynamics will once again either approach a stable limit cycle or a stable focus.

In support of this conjecture, we have been able to carry through explicit computations for the case of the two-dimensional cyclic attractor using the following proposition.

Proposition 1. ${ }^{(17)}$ Suppose $x_{0} \geqslant 0$ and $x_{i+1}=g\left(x_{i}\right)$ for $i=0,1,2, \ldots$, where $g$ is continuous on $[0, \infty)$, twice-differentiable on $(0, \infty)$ and satisfies the following properties:

- $g(0)=0$

- there is a $c_{0}>0$ such that $g\left(c_{0}\right) \leqslant c_{0}$;

- $g^{\prime}(x)>0$ for $x>0$;

- $g^{\prime \prime}(x)<0$ for $x>0$.

If $g^{\prime}(0) \leqslant 1$ (the derivative defined by a right-hand limit, or else $g$ smoothly extended below 0 ) then $\lim _{i \rightarrow \infty} x_{i}=0$, but if $g^{\prime}(0)>1$ then there is a unique positive fixed point that is attracting for all $x_{0}>0$.

Proof. Let $f(x)=g(x)-x$. Note that $f^{\prime}(x)=g^{\prime}(x)-1, f(0)=0$ and $f\left(c_{0}\right) \leqslant 0$.

Suppose first that $g^{\prime}(0) \leqslant 1$, so $f^{\prime}(0) \leqslant 0$. Since $f^{\prime \prime}(x)<0$, this implies that $f^{\prime}(x)<0$ and so $f(x)<0$ for $x>0$. Thus, $g(x)<x$ for all $x>0$, so the sequence $x_{i}$ converges to 0 for any $x_{0}>0$ (or trivially if $x_{0}=0$ ).

Now suppose that $g^{\prime}(0)>1$, so $f^{\prime}(0)>0$. Then $f$ is initially increasing so there is a point $c_{1} \in\left(0, c_{0}\right)$ with $f\left(c_{1}\right)>0$. Since $f\left(c_{0}\right) \leqslant 0$, there is also a point, $c_{2} \in\left(c_{1}, c_{0}\right]$ such that $f\left(c_{2}\right)=0$. This fixed point is unique since $f^{\prime \prime}(x)<0$ for $x>0$. Clearly, for $x \in\left(0, c_{2}\right)$ we have $f(x)>0$ so $g(x)>x$. Also, since $g$ is increasing, we have $g(x)<g\left(c_{2}\right)=c_{2}$. Thus, $x_{i}$ converges to $c_{2}$. Similarly, for $x \in\left(c_{2}, \infty\right)$ we have $f(x)<0$ so $g(x)<x$ but also $g(x)>$ $c_{2}$, so $x_{i}$ again converges to $c_{2}$.

For the two-dimensional cyclic attractor (i.e., Eq. (3) with $N=2$ and focal points chosen so that there is one entry boundary and one exit boundary to each quadrant), we can show that the return map on the positive $x_{1}$ axis has all the properties of $g$ in the Proposition, and that $g^{\prime}(0)$ depends on the focal point coordinates, but not the time constants. In particular, if the truth table is: 


\begin{tabular}{rrrr}
\hline orthant $(\mathbf{X})$ & focal pt. (f) \\
\hline 0 & 0 & $a_{1}$ & $-b_{1}$ \\
0 & 1 & $-a_{2}$ & $-b_{2}$ \\
1 & 0 & $a_{3}$ & $b_{3}$ \\
1 & 1 & $-a_{4}$ & $b_{4}$ \\
\hline
\end{tabular}

with all constants $a_{i}$ and $b_{i}$ positive, then the mappings starting from the positive $x_{1}$ axis to each of the subsequent axes going counterclockwise around the phase plane and returning to the positive $x_{1}$ axis are given by:

$$
\begin{aligned}
& M_{1}(x)=b_{4} \tau_{2}\left[1-\left(1+\frac{x}{a_{4} \tau_{1}}\right)^{-\tau_{1} / \tau_{2}}\right], \\
& M_{2}(x)=a_{2} \tau_{1}\left[-1+\left(1+\frac{x}{b_{2} \tau_{2}}\right)^{-\tau_{2} / \tau_{1}}\right], \\
& M_{3}(x)=b_{1} \tau_{2}\left[-1+\left(1-\frac{x}{a_{1} \tau_{1}}\right)^{-\tau_{1} / \tau_{2}}\right], \\
& M_{4}(x)=a_{3} \tau_{1}\left[1-\left(1-\frac{x}{b_{3} \tau_{2}}\right)^{-\tau_{2} / \tau_{1}}\right]
\end{aligned}
$$

so that the composite return map is $M(x)=M_{4}\left(M_{3}\left(M_{2}\left(M_{1}(x)\right)\right)\right)$. It is then tedious but straightforward to verify that the properties of $g$ in the proposition are satisfied by $M$, with $c_{0}=\max \left\{b_{4} \tau_{2}, a_{2} \tau_{1}, b_{1} \tau_{2}, a_{3} \tau_{1}\right\}$. A further calculation shows that

$$
M^{\prime}(0)=\frac{b_{1} a_{2} a_{3} b_{4}}{a_{1} b_{2} b_{3} a_{4}},
$$

so that $M^{\prime}(0)>1$ and there exists a stable limit cycle if $b_{1} a_{2} a_{3} b_{4}>$ $a_{1} b_{2} b_{3} a_{4}$. Note, however, that if there is no self input in the network, then necessarily $a_{1}=a_{3}, b_{1}=b_{2}, a_{2}=a_{4}$, and $b_{3}=b_{4}$, so that $M^{\prime}(0)=1$ and the origin is a stable focus, regardless of the time constants. Stability and existence of cycles in higher dimensions are more difficult to demonstrate when the time constants are unequal.

Poincaré maps can also be used in computer-aided analysis of chaotic dynamics in these networks. Two different approaches are possible. It is sometimes possible to demonstrate that the unstable manifold of a fixed point in the Poincare map intersects the stable manifold. This heteroclinic crossing does ensure that there will be a chaotic trajectory, but 
does not guarantee its stability. ${ }^{(18)}$ In some cases, it is possible to go much further. ${ }^{(20)}$ By analyzing the return maps to a given threshold hyperplane, in selected cases it has been possible to prove that there can be no stable fixed points given any periodic path that returns to a given threshold hyperplane so that the dynamics must either be quasiperiodic or chaotic. However, this method has only been successfully applied to four-dimensional networks and has only been demonstrated in particular cases. There is not a general method at present.

\subsection{Symbolic Dynamics and Topological Entropy}

Symbolic methods have played a prominent role in the study of dynamical systems. ${ }^{(31,32)}$ Because the trajectories in the dynamical systems considered here have a natural symbolic representation based on the directed graph on the hypercube, concepts from symbolic dynamics are applicable. In this section we discuss the dynamical properties of our network from a perspective of symbolic dynamics using classical results and also indicate some novel directions for further analysis.

Assume that the itinerary of the trajectory of a dynamical system $Y$ is represented by a sequence of symbols, called the alphabet. The number of letters in the alphabet is called the size of the alphabet. Denote the set of different blocks (i.e., symbol sequences) of length $n$ in a partial sequence of length $k$ in the dynamical system $Y$ by $B_{n}^{k}(Y)$ and the number of elements in this set by $\left|B_{n}^{k}(Y)\right| . B_{n}(Y)$ is the set of all blocks of length $n$ as $k \rightarrow \infty$, and $\left|B_{n}(Y)\right|=\lim _{k \rightarrow \infty}\left|B_{n}^{k}(Y)\right|$. One of the ways to characterize the complexity of a dynamical system $Y$ is by the topological entropy, which is

$$
h(Y)=\lim _{n \rightarrow \infty} \frac{1}{n} \log \left|B_{n}(Y)\right|
$$

For the dynamical system in Fig. 3(c), a natural choice for the alphabet is the set of vertices of the 5-cube. The trajectory is thus composed of a sequence of integers from the set $\{0,1,2, \ldots, 31\}$ corresponding to the decimal representation of the Boolean states. Based on the truth table for the network in Table II, we can place an upper limit on $\left|B_{n}^{k}(Y)\right|$. The adjacency matrix $W$ for this network is a $32 \times 32$ matrix where the elements represent the allowed transitions in the differential equation. Recall that $w_{i j}=1$ if there is a directed edge from vertex $i$ to vertex $j$ in the associated hypercube representation; otherwise $w_{i j}=0$. The total number of allowed paths of length $n$ from vertex $i$ to vertex $j$ in a Markov process based solely on these allowed single-step transitions is given by $w_{i j}^{(n)}$, 
the $(i j)$ th element of the matrix $W^{n}$. It follows from the Perron-Frobenius theorem that

$$
\left|B_{n}(Y)\right|=\Sigma_{i j} w_{i j}^{(n)} \leqslant d_{0} \lambda^{n},
$$

where $d_{0}$ is a positive constant, and $\lambda$ is the leading eigenvalue of the matrix $W .^{(31,32)}$ Consequently, the topological entropy is $h(Y) \leqslant \log \lambda$.

This computation counts all paths that are allowed by the truth table. However, in the differential equation it is possible that not all allowed transitions between vertices will actually be found, or not all allowed sequences of transitions. Thus, the above computation places an upper limit on the number of paths. In order to investigate the applicability of this theoretical result to the actual network, we numerically compute the value of $\left|B_{n}^{k}\right|$, for Eq. 3 with focal points defined by Table II and the derived parameters for the electronic network in Section 3, and compare this with the upper limit computed using Eq. (10). Figure 4 shows $\log _{2}\left|B_{n}^{k}\right|$ for $n=5,10$, and 15 , as a function of the number of switch times for the differential equation, panel (a), and the number of iterations for the Markov process based on the adjacency matrix defined by Table II, panel (b). For the symbol sequence generated by the differential equation, $\left|B_{n}\right|$ appears to converge to a value much less than the theoretical maximum, whereas in the equivalent stochastic process, $\left|B_{n}\right|$ appears to be converging to the theoretical limit, as it must. This point is further illustrated in Fig. 4(c) which gives $\left|B_{n}^{20}\right|$, for both the differential equation and the Markov process and compares these with the theoretical maximum. The leading eigenvalue of the adjacency matrix is $\lambda \approx 2.1935$, and so the topological entropy is $\log _{2}(\lambda) \approx 1.1332$ - which is the slope of the solid line in Fig. 4(c). It is difficult to estimate the topological entropy of the differential equation. Our numerical simulations suggest that it is considerably lower than that of the Markov process. Nevertheless, the topological entropy of the Markov process gives us a strict bound on the topological entropy of Eq. (3), and this bound can be computed based only on the logical structure of the system, without actually integrating the equation.

\subsection{Symbolic Dynamics of the Poincaré Map}

Although the representation of each vertex as a distinct symbol seems natural in the context of classical approaches in symbolic dynamics, other symbolic representations can also be useful for the current class of networks. ${ }^{(15,21)}$

First, we need several definitions. The coordinate sequence is the sequence of integers giving the logical coordinates (i.e., the indices of the 

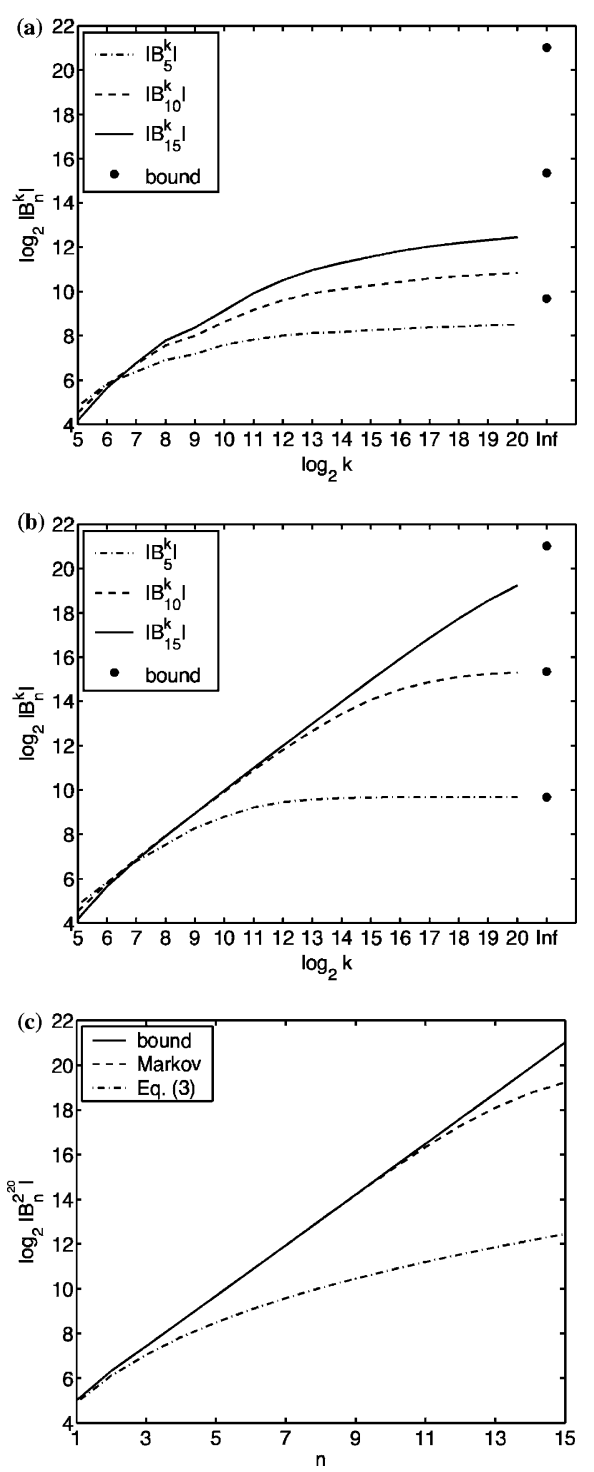

Fig. 4. Symbolic dynamics using the set of vertices of the 5-cube as the alphabet. (a) The number of unique blocks of length $n$ as a function of the number of switch times, $k$, for the differential equation with the truth table in Table II. (b) The number of unique blocks of length $n$ as a function of the number of iterations, $k$, for the Markov process defined by the adjacency matrix associated with the truth table in Table II. (c) The number of unique blocks of length $\mathrm{n}$ as a function of $\mathrm{n}$ following $k=2^{20}$ iterations in the Markov process and switch times in the differential equation with the truth table in Table II. The limiting value of the slope is given by the leading eigenvalue of the adjacency matrix. 
variables) that change as a path is traversed. For example, for the fivedimensional negative feedback cycle in Table I and Fig. 2 the coordinate sequence is $1234512345 \ldots$ The coordinate sequence is a compact way of representing a cycle, helping to clarify its symmetry properties. However, the coordinate sequence can be the same even for biological systems with different structures. Consider a feedback loop with three elements each of which only activates or inhibits the next in sequence. A loop with two activating links and one inhibitory link ${ }^{(14,15,19)}$ and a loop with three inhibitory links ${ }^{(4)}$ would both be associated with a cyclic attractor on the 3-cube and have the coordinate sequence 123123 , perhaps following permutation of the names of the elements.

A simple cycle is a cycle in which no vertex of the hypercube appears more than once. A simple cycle of length $L$ can be identified by the following criteria applied to the coordinate sequence: (i) $L$ is even; (ii) for any sequence of consecutive steps of length shorter than $L$, at least one coordinate must appear an odd number of times; (iii) In the coordinate sequence for the cycle of length $L$, each coordinate appears an even number of times. It follows that for dynamics on an $N$-cube, $4 \leqslant L \leqslant 2^{N}$. A complex cycle is any cycle that is not a simple cycle. In the directed 5-cube for our complex network, there are 35709 simple cycles including several Hamiltonian cycles.

The dynamical significance of simple and complex cycles is that they may be represented by a return map defined over a domain on the threshold hyperplane associated with an edge between two of the vertices on the cycle. In an earlier paper, ${ }^{(21)}$ we analyzed four-dimensional chaotic systems in Eq. (3) when all time constants were equal. For any choice of starting threshold hyperplane, we may consider each different (simple or complex) cycle arising in a return map to that hyperplane as a letter in an alphabet. Thus, each letter in such an alphabet corresponds to a block of vertex symbols in the alphabet of the previous subsection. A trajectory then corresponds to a sequence of letters representing consecutive return cycles. In general, we expect that the set of allowed sequences will be restricted. For example, in a previous publication ${ }^{(21)}$ we found that for a particular four-dimensional differential equation, we only needed two letters $\mathcal{C}$ and $\mathcal{D}$ since there were only two return cycles to a designated Poincare section. Further, the subsequence $\mathcal{C C}$ was forbidden. Thus, the dynamical system could be represented by the classical golden mean shift. ${ }^{(31,32)}$ We also described bifurcations that occurred as a function of the focal points. There were two types of bifurcation. In one, the numbers of consecutive allowed symbols could change. For example, we could have $\mathcal{C C}$, but not more than two consecutive appearances of $\mathcal{C}$. 
Another type of bifurcation requires the introduction of new symbols since the returns to the same Poincare section could follow additional paths through the hypercube so that the size of the alphabet increased to four symbols. The change in the qualitative properties of the chaotic dynamics here may be similar to the sudden qualitative changes called "crises" in other chaotic systems. ${ }^{(33)}$ All these bifurcations will lead to changes in the topological entropy of the dynamical system. These ideas have a correspondence in the theory of formal languages ${ }^{(34)}$ which have been discussed previously. ${ }^{(21)}$

The dynamics in the circuit and differential equations shown in Fig. 3 are more complex than the ones in ref. 21. We now consider a new way to characterize the complexity of the dynamics by plotting the size of the alphabet needed to characterize the return maps to the threshold hyperplane that is represented by the edge of the hypercube lying between vertices 00110 and 10110, in Fig. 5(a). Figure 5(b) shows the length distribution of the return map coordinate sequences found numerically after $2^{20}$ switch times. The longest cycle found had 202 switches.

The count in Fig. 5(a) is drawn as a function of the number of iterations for up to $2^{20}$ switch times. The number of return maps appears to be steadily increasing. To give an idea of the structure of the coordinate sequences, we next list three coordinate sequences, of lengths 58, 54 and 38 switches, respectively, starting from the vertex 00110 that are computed numerically during the complex dynamics displayed in Fig. 3(c).

1545121315[1212][1212][121424][124124][124124]32[4242][4242][4242][4242]4252

1545121315[1212][1212][121424][124124]32[421412][421412][421412]4252

$1545121315[1212][124124] 32[421412][425452] 4252$

A schematic representation of these three sequences is given in Fig. 6. The coordinate sequences in square brackets represent simple cycles. (Notice that there is not a unique way to parse these simple cycles and we provide one way of parsing). If we eliminate the simple cycles in square brackets from each example, we end up with the same coordinate sequence. We say that all coordinate sequences that can be brought into correspondence in this fashion, by continuing to eliminate simple cycles until the same simple cycle remains, are members of the same family. Even though the size of the alphabet characterizing the return map to a Poincaré section may be infinite, these will fall into a finite number of families that is bounded by the number of simple cycles containing the edge corresponding to the Poincare section. However, the coordinate sequence remaining after elimination of simple cycles is not necessarily unique. For 

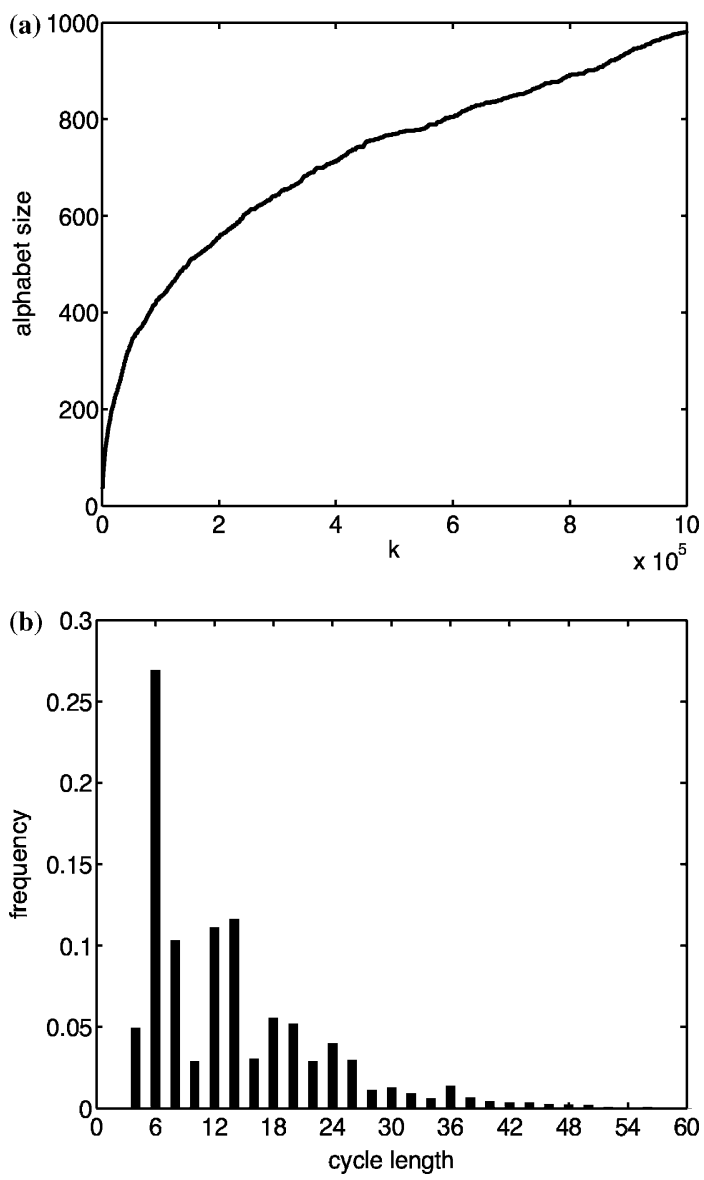

Fig. 5. Symbolic representation of complex dynamics. (a) The number of distinct letters (each representing a cycle) as a function of the number of switch times for the return to the boundary between orthants 00110 and 10110 in the differential equation with the parameters determined in the text. (b) The distribution of cycle lengths, i.e., numbers of vertices on each cycle. These are based on the complex network with optimized parameters, and $2^{20}$ switch times.

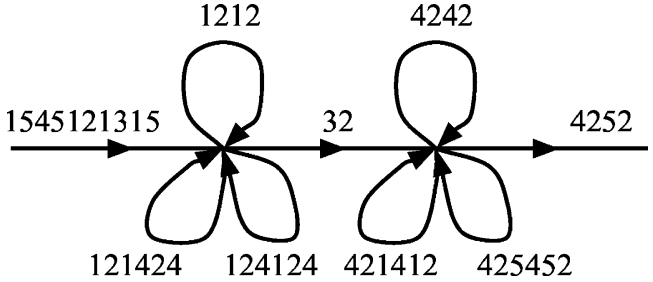

Fig. 6. Schematic representation of a family of coordinate sequences. 
example, the coordinate sequence 514142423543 can be simplified in two different ways:

$5[1414] 2423543 \rightarrow 52423543$ or $5141[4242] 3543 \rightarrow 51413543$.

Thus, a single coordinate sequence can belong to more than one family.

In Fig. 6, there are two vertices at which we have numerically found three different simple cycles. We define a secondary simple cycle as a simple cycle that is encountered as part of a return cycle, but that does not pass through the starting hyperplane (corresponding to an edge between two vertices). Further, the secondary simple cycles are traversed different numbers of times in these three examples. Based on our earlier studies in which we found that some simple cycles could be traversed an arbitrary number of times (as a consequence of heteroclinic crossings) we believe that in this network, there are situations in which one or more secondary simple cycles, that might be represented as a loop on a path as in Fig. 6, can be traversed an arbitrary number of times, and thus there will be an infinite number of maps that would describe the return to the starting threshold hyperplane. The numbers and characteristics of the return maps to different threshold hyperplanes will be different. For the following two propositions, we assume that we have a dynamical system in which all transients have dissipated so that the dynamics are on an asymptotic attractor.

Proposition 2. The size of the alphabet characterizing the return map to a threshold hyperplane on a trajectory is infinite if and only if there is a secondary cycle that can be traversed an arbitrary number of times.

Proof. If there is no secondary cycle, then the alphabet is composed of one letter for each simple cycle from the starting hyperplane (because on each, the sequence of vertices is fixed), and the number of such cycles is finite since the number of vertices is finite. If there are secondary cycles, but there is a maximum number of consecutive traversals of any such secondary cycle, then the alphabet is still bounded. If there is a secondary cycle that can be traversed an arbitrary number of times, then an infinite number of different return cycles is possible and therefore an infinite alphabet is required.

Proposition 3. In a differential equation described by Eq. (3), if for any threshold hyperplane traversed in a trajectory, the size of the alphabet needed to characterize the return map is infinite, then the dynamics are aperiodic.

Proof. Suppose the attractor is periodic; then the alphabet needed for one period, which is finite, suffices for infinite trajectories. Therefore, 
if there does not exist a finite alphabet for a return map regardless of the choice of starting threshold hyperplane (or graph edge), then the attractor cannot be periodic.

Note that arbitrarily long transients before convergence to an attractor can also require an infinite alphabet from some threshold hyperplane. The proposition refers to the situation on the attractor.

Notice also that if a trajectory has at least two simple cycles that can be traversed an arbitrary number of times, and these two simple cycles do not share any common edges, then independent of the choice of the threshold hyperplane, the size of the alphabet characterizing the return map must be infinite.

From Propositions 2 and 3 we conclude that:

Theorem 4. A necessary condition for chaotic dynamics in Eq. (3) is that there exists a complex cycle in the corresponding directed graph on the $N$-cube.

\section{CONCLUSIONS}

This paper raises issues that relate the qualitative dynamics in biological systems to the underlying logical structure as well as the quantitative values of the parameters in the system. The class of equations that we analyze here is notable for its preservation of a logical structure in a piecewise-linear ordinary differential equation. This class of equations enables mathematical analyses that incorporate traditional methods of nonlinear dynamics, such as analysis of stability of fixed points on return maps, as well as discrete methods, such as enumeration of the different return maps. Despite the results obtained so far towards predicting the qualitative features of the dynamics based on the logical structure, we still do not know how to identify networks that can display chaos for some choice of parameter values based on their logical structure. The analysis of the symbolic dynamics during chaotic dynamics raises intriguing theoretical questions concerning the connections between different symbolic representations of dynamical systems.

The potential relevance of these methods to real biological networks is not yet clear. Our study of chaotic dynamics in these networks opens the question of whether real genetic networks might display chaotic dynamics or might have evolved in a fashion to favor or suppress chaos, e.g., see ref. 7. Certainly, real systems differ from the equations here in many ways, including variable thresholds for different control tasks, continuous rather than step function control, stochastic effects, and time delays. Our comparison of a real electronic network with a differential 
equation demonstrates that the qualitative features of the dynamics can be preserved even though quantitative aspects of the dynamics are changed. Independent of its relevance to biology, this class of networks displays extraordinarily rich dynamics that are amenable to theoretical analysis, but are still barely understood.

\section{ACKNOWLEDGMENT}

This research has been supported by grants from NSERC, and the National Research Resource for Complex Physiologic Signals. T. Perkins thanks the National Science Foundation for support under a grant awarded in 2002. Any opinions, findings, and conclusions or recommendations expressed in this publication are those of the author(s) and do not necessarily reflect the views of the National Science Foundation. The electronic circuit was originally constructed in the laboratory of J. J. Collins, who we thank for support and discussion during the earlier phases of this project.

\section{REFERENCES}

1. B. Alberts, A. Johnson, J. Lewis, et al. Molecular Biology of the Cell, fourth edition (Garland Science, New York, 2002).

2. J. Monod and F. Jacob, General conclusions: Teleonomic mechanisms in cellular metabolism, growth, and differentiation, Cold Spring Harb. Symp. Quant. Biol. 26:389-401 (1961).

3. T. S. Gardner, C. R. Cantor, and J. J. Collins, Construction of a genetic toggle switch in Escherichia coli, Nature 403:339-342 (2000).

4. M. B. Elowitz and S. Leibler, A synthetic oscillatory network of transcriptional regulators, Nature 403:335-338 (2000).

5. J. Hasty, D. McMillen D, and J. J. Collins, Engineered gene circuits, Nature 420:224-230 (2002).

6. S. A. Kauffman, Metabolic stability and epigenesis in randomly constructed genetic networks, J. Theor. Biol. 22:437-467 (1969).

7. S. A. Kauffman, Origins of Order: Self-Organization and Selection in Evolution (Oxford University Press, Oxford, 1993).

8. U. Bastolla and G. Parisi, A numerical study of the critical line of Kauffman networks, J. Theor. Biol. 187:117-133 (1997).

9. S. Bilke and F. Sjunnesson, Stability of the Kauffman model, Phys. Rev. E 65:016129 (2001).

10. B. Derrida and Y. Pomeau, Random networks of automata - a simple annealed approximation, Europhys. Lett. 1:45-49 (1986).

11. H. Flyvbjerg, An order parameter for networks of automata, J. Phys. A: Math. Gen. 21:L955-L960 (1988).

12. X. Qu, M. Aldana, and L.P. Kadanoff, Numerical and theoretical studies of noise effects in the Kauffman model, J. Stat. Phys. 109:967-985 (2002). 
13. L. Glass and S. A. Kauffman, The logical analysis of continuous, nonlinear biochemical control networks, J. Theor. Biol. 39:103-129 (1973).

14. L. Glass, Combinatorial and topological methods in nonlinear chemical kinetics, J. Chem. Phys. 63:1325 (1975).

15. L. Glass, Combinatorial aspects of dynamics in biological systems. in Statistical Mechanics and Statistical Methods in Theory and Application, U. Landman, ed. (Plenum, New York, 1977), pp. 585-611.

16. L. Glass and J. S. Pasternack, Stable oscillations in mathematical models of biological control systems, J. Math. Biol. 6:207-223 (1978).

17. L. Glass, Boolean and continuous models for the generation of biological rhythms. in Dynamical Systems and Cellular Automata. J. Demongeot, E. Goles, M. Tchuente, eds. (Academic Press, London, 1985) pp. 197-206.

18. T. Mestl, C. Lemay, and L. Glass, Chaos in high dimensional neural and gene networks, Physica D 98:33-52 (1996).

19. R. Edwards and L. Glass, Combinatorial explosion in model gene networks, Chaos 10:691-704 (2000).

20. R. Edwards, Chaos in neural and gene networks with hard switching, Diff. Eq. Dyn. Sys. 9:187-220 (2001).

21. R. Edwards, H. T. Siegelmann, K. Aziza, and L. Glass, Symbolic dynamics and computation in model gene networks, Chaos 11:160-169 (2001).

22. T. J. Perkins, M. T. Hallett, and L. Glass, Inferring models of gene expression dynamics, J. Theor. Biol. 230:289-299 (2004).

23. R. Edwards, Analysis of continuous time switching networks, Physica D 146:165-199 (2000).

24. R. Edwards and L. Glass, A calculus for relating the dynamics and structure of complex biological networks, in Adventures in Chemical Physics: A Special Volume of Advances in Chemical Physics, Vol. 132, edited by R. S. Berry and J. Jortner (John Wiley \& Sons, Inc. Hoboken, NJ, 2006) pp. 151-178.

25. J. P. Mason, P. S. Linsay, J. J. Collins, and L. Glass, Evolving complex dynamics in electronic models of genetic networks, Chaos 14:707-715 (2004).

26. B. C. Goodwin, Analytical Physiology of Cells and Developing Organisms (Academic Press, London, 1976).

27. R. Thomas and R. D’Ari, Biological Feedback (CRC Press, Boca Raton, 1990).

28. H. H. McAdams and L. Shapiro, Circuit simulation of genetic networks, Science 269:650-656 (1995).

29. H. De Jong, J. L. Gouze, C. Hernandez C et al. Qualitative simulation of genetic regulatory networks using piecewise-linear models, Bull. Math. Biol. 66:301-340 (2004).

30. S. Muroga, Logic Design and Switching Theory (John Wiley, New York, 1979).

31. D. Lind and B. Marcus, An Introduction to Symbolic Dynamics and Coding (Cambridge University Press, Cambridge, 1995).

32. M. Brin and G. Stuck, Introduction to Dynamical Systems (Cambridge University Press, Cambridge, 2002).

33. C. Grebogi, E. Ott, and J. A. Yorke, Chaotic attractors in crisis, Phys. Rev. Lett. 48:1507-1510 (1982).

34. M. Sipser, Introduction to the Theory of Computation (Boston, PWS Publishers, 1997). 